\title{
Current Status of Deep Brain Stimulation for Alzheimer's Disease: From Chance Observation to Clinical Trials
}

\author{
Darrin J. LeE ${ }^{1}$ and Andres M. Lozano ${ }^{2}$ \\ ${ }^{1}$ Department of Neurological Surgery, University of Southern California, Los Angeles, \\ California 90033, USA \\ ${ }^{2}$ Division of Neurosurgery, Department of Surgery, Toronto Western Hospital and University of Toronto, \\ Krembil Brain Institute, Toronto, Ontario MST 258, Canada \\ Correspondence: andres.lozano@uhnresearch.ca
}

\begin{abstract}
Alzheimer's disease (AD) is a neurodegenerative disorder that results in significant memory impairment and cognitive decline. Current medical treatment is aimed at treating AD symptoms but does not alter the disease course. The use of deep brain stimulation (DBS) for the treatment of $\mathrm{AD}$ is in its nascent phase. Here, we describe the evolution of DBS as a potential treatment modality for $\mathrm{AD}$, including previous and current trials, as well as the behavioral and histological preclinical data that help to better understand and inform future clinical trials. As such, a phase 3 clinical trial studying the effects of forniceal DBS for $\mathrm{AD}$ is currently underway.
\end{abstract}

Alzheimer's disease (AD) is a progressive neurodegenerative disease that results in cognitive dysfunction. It has several hallmark pathological features including $\beta$-amyloid plaques, neurofibrillary tangles, and neuronal cell death that lead to functional deterioration (Schneider and Sari 2014). Although there are currently no treatments to reverse or even slow the progression of $\mathrm{AD}$, medications are aimed at temporarily reducing cognitive dysfunction, such as memory loss and confusion. Current medications primarily target increasing acetylcholine (cholinesterase inhibitors) or blocking glutamate (NMDA receptor antagonists).

In $\mathrm{AD}$, morphological and structural changes occur in the brain with widespread involvement of the hippocampus, association cortices, amygdala, nucleus basalis of Meynert (nbM), and other structures. Alterations in the hippocampus and entorhinal cortex (EC) occur before cognitive symptoms and correlate with the severity of the disease (Risacher et al. 2009). In addition to structural changes, there is aberrant functional activity. In early $\mathrm{AD}$, for example, there is evidence for a reduction in glucose metabolism in the temporal lobe and posterior cingulate gyrus (Smith et al. 1992; Minoshima et al. 1997).

There is increasing evidence that $\mathrm{AD}$ is associated with network dysfunction. More specifically, there are perturbations in the circuit of Papez (Sperling et al. 2010), default mode network (Greicius et al. 2004), and salience network (Zhou et al. 2010). In AD, there is evidence for reduced functional connectivity in the circuit of Papez ( $\mathrm{Li}$ et al. 2014) and default mode network but increased connectivity in the salience network (Zhou et al. 2010). These alterations in connectivity are associated with deficits in working memory and cognition.
Deep brain stimulation (DBS) is an electrical form of neuromodulation that enables for spatially and temporally specific stimulation. Historically, this treatment modality has been used in movement disorders, such as Parkinson's disease, essential tremor, and dystonia; however, more recently, it has been explored in cognitive and psychiatric disorders, including obsessive-compulsive disorder, depression, and epilepsy. Here, we describe the evolution of our translational and clinical research using DBS for $\mathrm{AD}$.

\section{ENGAGING THE CIRCUIT OF PAPEZ WITH DEEP BRAIN STIMULATION}

During bilateral hypothalamic DBS surgery for obesity, we serendipitously discovered that as an electrode was being advanced toward the hypothalamus, periforniceal stimulation $(3.0 \mathrm{~V}, 60 \mu \mathrm{sec}$ pulse width, and $130 \mathrm{~Hz})$ triggered autobiographical memories intraoperatively (Hamani et al. 2008). After 3 wk of bilateral hypothalamic stimulation, using stimulation parameters that did not induce overt memory, sensory, or autonomic effects $(2.8 \mathrm{~V}$, $60 \mu \mathrm{sec}$ pulse width, and $130 \mathrm{~Hz}$ ), the patient showed improvements in neuropsychological assessments (California Verbal Learning Test and Spatial Associative Learning Test). Two months after implantation, the patient underwent a battery of memory tests with stimulation identified to elicit memories intraoperatively $(3.0 \mathrm{~V}, 60$ $\mu$ sec pulse width, and $130 \mathrm{~Hz}$ ). Here, stimulation enhanced hippocampal-dependent recollection but not familiarity-based recognition.

To confirm that stimulation was activating the Papez circuit, standardized low-resolution electromagnetic to-

(C) 2018 Lee and Lozano. This article is distributed under the terms of the Creative Commons Attribution-NonCommercial License, which permits reuse and redistribution, except for commercial purposes, provided that the original author and source are credited. 
mography was conducted after 1 mo of stimulation. During SLORETA, $3 \mathrm{~Hz}$ of unilateral stimulation activated the ipsilateral hippocampal and parahippocampal formations. Taken together, these findings suggested that it is possible to engage the circuit of Papez with forniceal DBS and enhance hippocampal-dependent memory.

\section{FORNICEAL DBS FOR AD}

The hallmark symptoms of $\mathrm{AD}$ are functional impairments in cognitive and memory functions. Given the potentially beneficial effects of stimulation on memory and activation of the Papez circuit, we proposed a six-patient phase 1 clinical trial using forniceal DBS in mild AD patients. In this study, patients received $1 \mathrm{yr}$ of continuous bilateral stimulation $(130 \mathrm{~Hz}, 3-3.5 \mathrm{~V}, 90 \mu \mathrm{sec}$ pulse width) (Laxton et al. 2010). At $12 \mathrm{mo}$, half the patients showed a slight worsening in the Alzheimer's Disease Assessment Scale, Cognitive subscale (ADAS-Cog), whereas the other half showed a mild improvement. Interestingly, the patient who had the most vivid intraoperative experiential recollections had the greatest improvement in ADAS-Cog scores. Moreover, there may have been a less than expected decline in Mini-Mental Status Exam (MMSE) scores over the 12-mo period.

After $1 \mathrm{yr}$, sLORETA of these six patients showed activation of memory circuits (frontal-temporal-parietalstriatal-thalamic and frontal-temporal-parietal-occipitalhippocampal networks) and the default mode network, including the hippocampus, parahippocampal gyrus, cingulate gyrus, and precuneus. AD is associated with decreased glucose metabolism in the temporal and parietal regions (Smith et al. 1992; Minoshima et al. 1997). Although anticholinergic medications have shown transient increases in glucose cerebral metabolism, 1 yr of DBS resulted in persisting increased temporal and parietal glucose metabolism in regions relatively spared in AD (Figs. 1 and 2; Laxton et al. 2010; Smith et al. 2012). Interestingly, increased metabolism was correlated with improvements in cognition, memory, and quality of life (Smith et al. 2012).

$\mathrm{AD}$ is characterized by atrophy in several brain structures. Of note, there is a correlation between site-specific volumetry (lower subiculum volume and fornix integrity) and cerebrospinal fluid biomarkers (decreased CSFA $\beta$ and tau levels) as well as associated lower cognitive scores (Tardif et al. 2018). In addition to activating the Papez circuit, we hypothesized that forniceal DBS may influence the progressive atrophy characteristic of AD. To our surprise, in the two patients who had the best clinical outcomes, there were bilateral increases in hippocampal volume after 1 yr of stimulation. Overall, hippocampal, fornix, and mammillary body atrophy was slower in the six DBS patients relative to age-matched AD controls, suggesting network-wide activation (Sankar et al. 2015).

Based on these findings, a 12-mo, double-blind, randomized controlled feasibility study was performed (ADvance trial). 42 patients with mild AD (Clinical Dementia Rating Sum of Boxes [CDR-SB] of 0.5 or 1) underwent bilateral forniceal DBS implantation (Lozano et al. 2016).

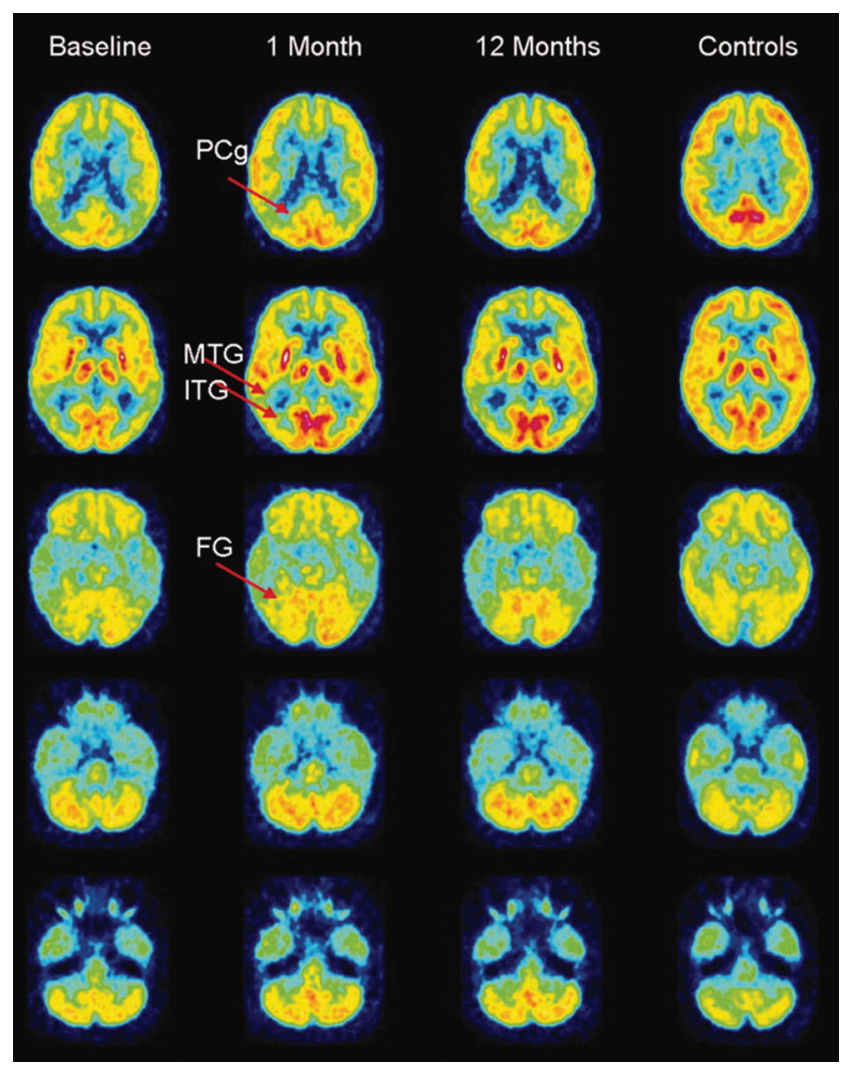

Figure 1. A composite positron emission tomography of the five Alzheimer's patients at baseline ( 1 mo before surgery), 1 mo after, and 12 mo after continuous bilateral forniceal/hypothalamic deep brain stimulation as well as six age-matched health controls. There was increased metabolism in the temporal, posterior cingulate, and parietal regions both at $1 \mathrm{mo}$ and sustained at $12 \mathrm{mo}$. The flame scale demonstrates fluorodeoxyglucose use per $100 \mathrm{~g}$ tissue/min, with red indicating the highest values and blue the lowest values. (MTG) Middle temporal gyrus, $(\mathrm{PCg})$ posterior cingulate gyrus, (ITG) inferior temporal gyrus, (FG) fusiform gyrus. (Reprinted from Laxton et al. 2010, with permission from John Wiley and Sons.) 


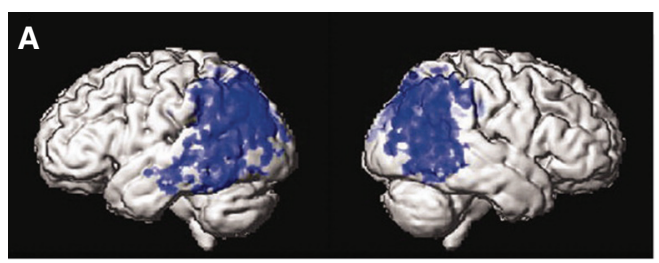

Decreased metabolism in $A D$ compared to Controls

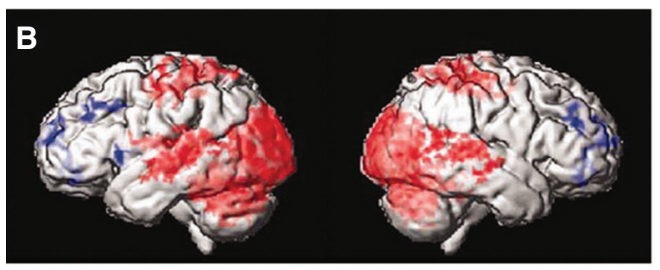

One month of DBS compared to baseline

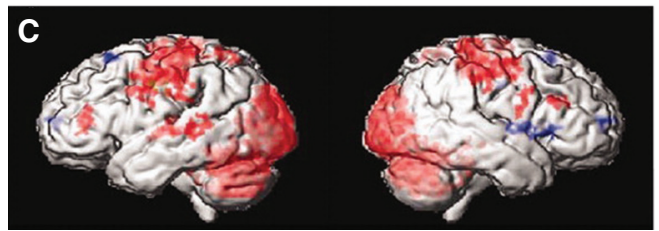

One year of DBS compared

to baseline

Figure 2. Voxel-wise comparisons of positron emission tomography of glucose metabolism. (A) Prior to DBS, AD patients show decreased metabolism compared to controls. $(B)$ One month of bilateral forniceal/hypothalamic DBS, the temporal regions (left middle and bilateral inferior temporal gyri), parietal cortical regions (bilateral fusiform gyri and superior parietal lobules, right precuneus, left posterior cingulate gyrus, and inferior parietal lobule), sensory and motor (left pre- and postcentral gyrus, bilateral lingual and bilateral cuneus gyri) and cerebellar regions (left medulla), and cerebellum (bilateral dentate and culmen) showed significant increases in metabolism. The anterior cortical areas (bilateral anterior cingulate, right medial and middle frontal and bilateral precentral gyri) and subcortical areas (left caudate and thalamus [medial dorsal nuclei]) showed decreased metabolism. (C) After 12 mo of DBS, these effects on metabolism were sustained. (Reprinted from Laxton et al. 2010, with permission from John Wiley and Sons.)

In this trial, patients were randomized to continuous bilateral stimulation $(130 \mathrm{~Hz}, 3-3.5 \mathrm{~V}, 90 \mu \mathrm{sec}$ pulse width) or sham stimulation for 12 mo. There were no significant differences between the two groups with respect to primary outcomes, such as ADAS-Cog 13 and CDR-SB scores, or secondary clinical outcomes (California Verbal Learning Test-Second Edition, Alzheimer's Disease Cooperative Study Activities of Daily Living Scale and Neuropsychiatric Inventory).

Although there was no significant difference in primary or secondary outcomes, a post hoc analysis showed that younger patients receiving stimulation $(<65 \mathrm{yr})$ had a significant worsening in ADAS-Cog-13 and CDR-SB scores compared to sham stimulation. In contrast, older patients receiving stimulation ( $\geq 65 \mathrm{yr}$ ) appeared to have less decline than sham stimulation patients.

Interestingly, the $<65$-yr-old group showed further decreased PET cerebral glucose metabolism after $1 \mathrm{yr}$ of stimulation within regions typically affected in AD, such as the temporal and parietal regions. In contrast, the $\geq 65$ yr-old cohort showed increased metabolism within the temporal and parietal regions with stimulation after 6 mo (Lozano et al. 2016). Additionally, stimulation also increased metabolism in regions that are relatively spared in $\mathrm{AD}$, such as sensory and motor cortex as well as the cerebellum.

After the first year of randomization, all patients underwent bilateral forniceal stimulation. Although the study showed that forniceal DBS had a favorable safety profile, the study was not powered to determine a clinical benefit. However, it suggested a possible benefit in the older pop- ulation ( $>65 \mathrm{yr}$ ) (Leoutsakos et al. 2018). As such, we are in the process of enrolling a phase 3 multicenter clinical trial across North America and Europe to evaluate the potential benefits of bilateral forniceal DBS in the older cohort $(>65 \mathrm{yr})$. This randomized control trial plans to enroll 150 patients to either forniceal or no bilateral forniceal stimulation over the course of $12 \mathrm{mo}$.

\section{OTHER DBS TARGETS FOR AD}

Subsequent DBS trials have examined other targets including the nbM and the ventral capsule/ventral striatum (VC/VS). The nbM is a group of cholinergic neurons in the substantia innominate of the basal forebrain that are particularly susceptible to neurodegeneration in AD (Arendt et al. 1983). Furthermore, nonhuman primate intermittent nbM DBS resulted in improved working memory that was dependent on cholinergic function (Blake et al. 2017). In a six-patient pilot study of nbM DBS, four of the six patients were considered responders (relatively stable ADAS-Cog and MMSE scores) (Kuhn et al. 2015). Stimulation parameters ranged from 10 to $20 \mathrm{~Hz}$ frequency, 90-150 $\mu \mathrm{sec}$ pulse width, and 2.0-4.5 V. Consistent with the forniceal DBS subgroup analysis, patients with greater fronto-parieto-temporal cortical thickness (i.e., less advanced atrophy) had a greater cognitive benefit from nbM DBS (Baldermann et al. 2018).

The VC/VS has projections to the dorsomedial and orbital prefrontal cortices, which are important in executive function. As such, VC/VS DBS was performed in three 
$\mathrm{AD}$ patients with less cognitive decline than age-matched controls over at least an 18-mo period. FDG-PET imaging was consistent with frontal cortical engagement (Scharre et al. 2018).

\section{MECHANISM OF DBS FOR AD}

Although forniceal DBS has been shown to functionally engage the hippocampus, the mechanism of DBS is still not completely understood. Rodent studies show that forniceal DBS can improve both hippocampal-dependent and hippocampal-independent memory (Zhang et al. 2015). Interestingly, there is evidence that the effects of forniceal DBS on memory are not dependent on the frequency of stimulation but are dependent on the current levels (Hescham et al. 2013). Based on the observation that two of the six patients had enlargement of their hippocampi (as opposed to expected atrophy) (Sankar et al. 2015), there have been a number of rodent studies that have shown that electrical stimulation of limbic structures can promote adult neurogenesis, including EC stimulation $(130 \mathrm{~Hz}, 90 \mu \mathrm{sec}$ pulse width, $50 \mu \mathrm{A})$ (Stone et al. 2011; Mann et al. 2018) and anterior thalamic nucleus stimulation (Toda et al. 2008; Encinas et al. 2011). Interestingly, forniceal DBS $(130 \mathrm{~Hz}, 90 \mu \mathrm{sec}$ pulse width, $2.5 \mathrm{~V}$ ) results in increased expression of neurotrophic factors (BDNF, VEGF) and markers of synaptic plasticity (GAP-43, synaptophysin, $\alpha$-synuclein [Gondard et al. 2015]).

The EC and thalamus have also been posited as potential DBS targets (Arrieta-Cruz et al. 2010). EC DBS has also been shown to improve behavior in $\mathrm{AD}$ rodent models. In a TgCRND8 (Tg) transgenic mouse model expressing human amyloid precursor protein harboring the Swedish and Indiana familial AD mutations, EC DBS rescued contextual fear and spatial memory as well as a decreased plaque load. Of note, the memory enhancement emerged gradually and was specific to hippocampal-dependent memory (Xia et al. 2017). Thalamic DBS has also been shown to improve hippocampal-dependent short-term memory in the $\mathrm{Tg}$ transgenic mouse as well (Arrieta-Cruz et al. 2010). Interestingly, a rodent study comparing EC, thalamic, and forniceal DBS showed that EC and forniceal DBS improved hippocampal-dependent memory more robustly than thalamic DBS. Moreover, EC and forniceal DBS also had beneficial effects on hippocampal-independent recognition memory. Importantly, EC, thalamic, and forniceal DBS did not result in increased anxiety or locomotor behaviors (Zhang et al. 2015).

There is also evidence that chronic EC DBS in a triple transgenic mouse model $(3 \times \mathrm{Tg}-\mathrm{AD})$ or chemically induced synaptic stimulation of an in vitro culture of the EC in the 3xTg-AD model can reduce the accumulation of pathological forms of Tau via autophagosomes and lysosomes (Akwa et al. 2018). Interestingly, chronic inhibition of synaptic activity increased tau accumulation. Additionally, EC DBS reduces $\beta$-amyloid plaques, CA-1 cellular $\beta$-amyloid-42 levels, and cortical and hippocampal tau (Mann et al. 2018).

\section{CONCLUSION}

Despite the fact that DBS for AD has had varying results, there is preclinical and clinical evidence that forniceal DBS engages networks important in memory and may affect AD-associated hippocampal atrophy. The phase 2 ADvance trial suggests that older patients may benefit from forniceal DBS, whereas younger patients do not have cognitive benefits. The upcoming phase 3 clinical trial involving an exclusively older patient cohort will help to determine the effectiveness of forniceal DBS on cognition and disease progression; however, it is important to understand why only a subset of patients would respond to treatment.

Further work is necessary to identify the effects of stimulation on sustained structural and functional changes and their potential relationship to treatment response. DBS stimulation parameters are currently informed by movement disorder pathology; however, engaging memory networks may need different stimulation parameters (i.e., theta, theta-burst stimulation). There is no evidence for network engagement and structural effects, suggesting the benefits of continuous stimulation. However, the ideal stimulation parameters for cognition may not require continuous stimulation. In fact, constant stimulation may interfere with normal cognition. As evidenced by variable responses to medications and stimulation, tailored treatments are necessary for individuals. Current DBS technology allows for responsive neurostimulation. Thus, identifying patient-specific electrophysiological biomarkers will be potentially important for tailoring stimulation.

The underlying mechanism of DBS for AD is still not known. Although DBS is able to target specific structures, with advances in technology, such as optogenetics, sonogenetics, and magnetogenetics, it is now possible to evaluate the effects of cell type-specific stimulation in a spatially and temporally relevant manner. Understanding the effects of stimulation on the underlying disease process will also help to inform stimulation parameters and targets. Similarly, identifying and creating better animal models will help with the difficulties in translating these findings to human clinical trials.

Taken together, DBS for AD remains a potentially promising new treatment for a devastating disease. Although it is safe in patients with $\mathrm{AD}$, future studies will help to determine its efficacy and potential as an adjunctive treatment modality.

\section{REFERENCES}

Akwa Y, Gondard E, Mann A, Capetillo-Zarate E, Alberdi E, Matute C, Marty S, Vaccari T, Lozano AM, Baulieu EE, et al. 2018. Synaptic activity protects against AD and FTDlike pathology via autophagic-lysosomal degradation. $\mathrm{Mol}$ Psychiatry 23: 1530-1540. doi:10.1038/mp.2017.142

Arendt T, Bigl V, Arendt A, Tennstedt A. 1983. Loss of neurons in the nucleus basalis of Meynert in Alzheimer's disease, paralysis agitans and Korsakoff's disease. Acta Neuropathol 61: 101-108. doi:10.1007/BF00697388

Arrieta-Cruz I, Pavlides C, Pasinetti GM. 2010. Deep brain stimulation in midline thalamic region facilitates synaptic transmis- 
sion and short term memory in a mouse model of Alzheimer's disease. Transl Neurosci 1: 188-194.

Baldermann JC, Hardenacke K, Hu X, Köster P, Horn A, Freund HJ, Zilles K, Sturm V, Visser-Vandewalle V, Jessen F, et al. 2018. Neuroanatomical characteristics associated with response to deep brain stimulation of the nucleus basalis of Meynert for Alzheimer's disease. Neuromodulation 21: 184190. doi:10.1111/ner.12626

Blake DT, Terry AV, Plagenhoef M, Constantinidis C, Liu R. 2017. Potential for intermittent stimulation of nucleus basalis of Meynert to impact treatment of Alzheimer's disease. Commun Integr Biol 10: e1389359. doi:10.1080/19420889.2017 .1389359

Encinas JM, Hamani C, Lozano AM, Enikolopov G. 2011. Neurogenic hippocampal targets of deep brain stimulation. J Comp Neurol 519: 6-20. doi:10.1002/cne.22503

Gondard E, Chau HN, Mann A, Tierney TS, Hamani C, Kalia SK, Lozano AM. 2015. Rapid modulation of protein expression in the rat hippocampus following deep brain stimulation of the fornix. Brain Stimul 8: 1058-1064. doi:10.1016/j.brs .2015.07.044

Greicius MD, Srivastava G, Reiss AL, Menon V. 2004. Defaultmode network activity distinguishes Alzheimer's disease from healthy aging: evidence from functional MRI. Proc Natl Acad Sci 101: 4637-4642. doi:10.1073/pnas.0308627101

Hamani C, McAndrews MP, Cohn M, Oh M, Zumsteg D, Shapiro CM, Wennberg RA, Lozano AM. 2008. Memory enhancement induced by hypothalamic/fornix deep brain stimulation. Ann Neurol 63: 119-123. doi:10.1002/ana.21295

Hescham S, Lim LW, Jahanshahi A, Steinbusch HW, Prickaerts J, Blokland A, Temel Y. 2013. Deep brain stimulation of the forniceal area enhances memory functions in experimental dementia: the role of stimulation parameters. Brain Stimul 6: 72-77. doi:10.1016/j.brs.2012.01.008

Kuhn J, Hardenacke K, Lenartz D, Gruendler T, Ullsperger M, Bartsch C, Mai JK, Zilles K, Bauer A, Matusch A, et al. 2015. Deep brain stimulation of the nucleus basalis of Meynert in Alzheimer's dementia. Mol Psychiatry 20: 353-360. doi: $10.1038 / \mathrm{mp} .2014 .32$

Laxton AW, Tang-Wai DF, McAndrews MP, Zumsteg D, Wennberg R, Keren R, Wherrett J, Naglie G, Hamani C, Smith GS, et al. 2010. A phase I trial of deep brain stimulation of memory circuits in Alzheimer's disease. Ann Neurol 68: 521-534. doi:10.1002/ana.22089

Leoutsakos JS, Yan H, Anderson WS, Asaad WF, Baltuch G, Burke A, Chakravarty MM, Drake KE, Foote KD, Fosdick L, et al. 2018. Deep brain stimulation targeting the fornix for mild Alzheimer Dementia (the ADvance trial): a two year follow-up including results of delayed activation. J Alzheimers Dis 64: 597-606. doi:10.3233/JAD-180121

Li W, Antuono PG, Xie C, Chen G, Jones JL, Ward BD, Singh SP, Franczak MB, Goveas JS, Li SJ. 2014. Aberrant functional connectivity in Papez circuit correlates with memory performance in cognitively intact middle-aged APOE4 carriers. Cortex 57: 167-176. doi:10.1016/j.cortex .2014 .04 .006

Lozano AM, Fosdick L, Chakravarty MM, Leoutsakos JM, Munro C, Oh E, Drake KE, Lyman CH, Rosenberg PB, Anderson WS, et al. 2016. A Phase II study of fornix deep brain stimulation in mild Alzheimer's disease. J Alzheimers Dis 54: $777-$ 787. doi:10.3233/JAD-160017

Mann A, Gondard E, Tampellini D, Milsted JAT, Marillac D, Hamani C, Kalia SK, Lozano AM. 2018. Chronic deep brain stimulation in an Alzheimer's disease mouse model enhances memory and reduces pathological hallmarks. Brain Stimul 11: 435-444. doi:10.1016/j.brs.2017.11.012

Minoshima S, Giordani B, Berent S, Frey KA, Foster NL, Kuhl DE. 1997. Metabolic reduction in the posterior cingulate cor- tex in very early Alzheimer's disease. Ann Neurol 42: 85-94. doi:10.1002/ana.410420114

Risacher SL, Saykin AJ, West JD, Shen L, Firpi HA, McDonald BC, Alzheimer's Disease Neuroimaging Initiative (ADNI). 2009. Baseline MRI predictors of conversion from MCI to probable AD in the ADNI cohort. Curr Alzheimer Res 6: 347-361. doi:10.2174/156720509788929273

Sankar T, Chakravarty MM, Bescos A, Lara M, Obuchi T, Laxton AW, McAndrews MP, Tang-Wai DF, Workman CI, Smith GS, et al. 2015. Deep brain stimulation influences brain structure in Alzheimer's disease. Brain Stimul 8: 645-654. doi:10.1016/j .brs.2014.11.020

Scharre DW, Weichart E, Nielson D, Zhang J, Agrawal P, Sederberg PB, Knopp MV, Rezai AR, Alzheimer's Disease Neuroimaging Initiative (ADNI). 2018. Deep brain stimulation of frontal lobe networks to treat Alzheimer's disease. $J$ Alzheimers Dis 62: 621-633. doi:10.3233/JAD-170082

Schneider AR, Sari Y. 2014. Therapeutic perspectives of drugs targeting Toll-like receptors based on immune physiopathology theory of Alzheimer's disease. CNS Neurol Disord Drug Targets 13: 909-920. doi:10.2174/1871527313666140711 093858

Smith GS, de Leon MJ, George AE, Kluger A, Volkow ND, McRae T, Golomb J, Ferris SH, Reisberg B, Ciaravino J, et al. 1992. Topography of cross-sectional and longitudinal glucose metabolic deficits in Alzheimer's disease. Pathophysiologic implications. Arch Neurol 49: 1142-1150. doi: 10.1001/archneur.1992.00530350056020

Smith GS, Laxton AW, Tang-Wai DF, McAndrews MP, Diaconescu AO, Workman CI, Lozano AM. 2012. Increased cerebral metabolism after 1 year of deep brain stimulation in Alzheimer disease. Arch Neurol 69: 1141-1148. doi:10.1001/archneurol .2012 .590

Sperling RA, Dickerson BC, Pihlajamaki M, Vannini P, LaViolette PS, Vitolo OV, Hedden T, Becker JA, Rentz DM, Selkoe DJ, et al. 2010. Functional alterations in memory networks in early Alzheimer's disease. Neuromolecular Med 12: 27-43. doi:10.1007/s12017-009-8109-7

Stone SS, Teixeira CM, DeVito LM, Zaslavsky K, Josselyn SA, Lozano AM, Frankland PW. 2011. Stimulation of entorhinal cortex promotes adult neurogenesis and facilitates spatial memory. J Neurosci 31: 13469-13484. doi:10.1523/ JNEUROSCI.3100-11.2011

Tardif CL, Devenyi GA, Amaral RSC, Pelleieux S, Poirier J, Rosa-Neto P, Breitner J, Chakravarty MM, PREVENT-AD Research Group. 2018. Regionally specific changes in the hippocampal circuitry accompany progression of cerebrospinal fluid biomarkers in preclinical Alzheimer's disease. Hum Brain Mapp 39: 971-984. doi:10.1002/hbm.23897

Toda H, Hamani C, Fawcett AP, Hutchison WD, Lozano AM. 2008. The regulation of adult rodent hippocampal neurogenesis by deep brain stimulation. J Neurosurg 108: 132-138. doi: $10.3171 / \mathrm{JNS} / 2008 / 108 / 01 / 0132$

Xia F, Yiu A, Stone SSD, Oh S, Lozano AM, Josselyn SA, Frankland PW. 2017. Entorhinal cortical deep brain stimulation rescues memory deficits in both young and old mice genetically engineered to model Alzheimer's disease. Neuropsychopharmacology 42: 2493-2503. doi:10.1038/npp. 2017.100

Zhang C, Hu WH, Wu DL, Zhang K, Zhang JG. 2015. Behavioral effects of deep brain stimulation of the anterior nucleus of thalamus, entorhinal cortex and fornix in a rat model of Alzheimer's disease. Chin Med $J$ (Engl) 128: 1190-1195. doi:10.4103/0366-6999.156114

Zhou J, Greicius MD, Gennatas ED, Growdon ME, Jang JY, Rabinovici GD, Kramer JH, Weiner M, Miller BL, Seeley WW. 2010. Divergent network connectivity changes in behavioural variant frontotemporal dementia and Alzheimer's disease. Brain 133: 1352-1367. doi:10.1093/brain/awq075 


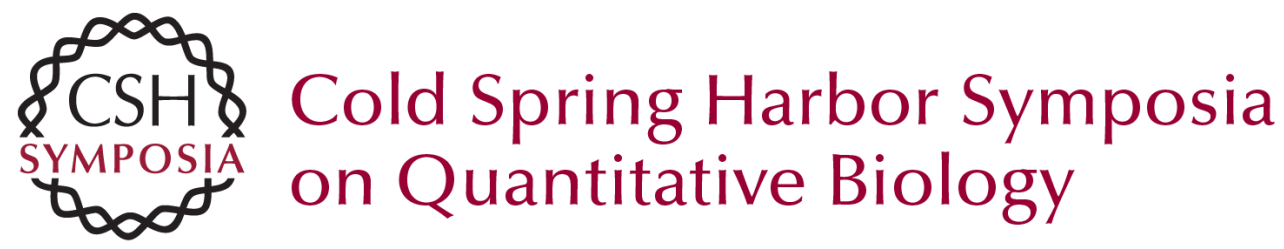

\section{Current Status of Deep Brain Stimulation for Alzheimer's Disease: From Chance Observation to Clinical Trials}

Darrin J. Lee and Andres M. Lozano

Cold Spring Harb Symp Quant Biol 2018 83: 201-205 originally published online March 8, 2019 Access the most recent version at doi:10.1101/sqb.2018.83.037440

References This article cites 30 articles, 2 of which can be accessed free at: http://symposium.cshlp.org/content/83/201.full.html\#ref-list-1

Creative This article is distributed under the terms of the

Commons http://creativecommons.org/licenses/by-nc/4.0/, which permits reuse and License redistribution, except for commercial purposes, provided that the original author and source are credited.

Email Alerting Receive free email alerts when new articles cite this article - sign up in Service the box at the top right corner of the article or click here. 